junction with this division of the book, as it contains a set of formulæ, deduced from Fourier's theorem, with the coefficients worked out, for calculating the harmonics (up to the IIth) of an alternating current waveform. The author states that in all curves actually met with in practice, the $13^{\text {th }}$ and higher harmonics can be neglected, as they are so small. This is, however, not true. It has been recently shown that in the E.M.F. curve of the alternators of the Glasgow Corporation Tramways, the I $3^{\text {th }}$ harmonic is one of the most important, and alternators may very well exist in which the $15^{\text {th }}$ and $17^{\text {th }}$ harmonics are the largest.

The last division, on polyphase currents, does not do more than show the general star and delta relationships, and contains a chapter on the measurement of three-phase power.

As stated above, the book will be mainly useful to practical engineers who desire to have at hand a volume which will help them out of mental entanglements which arise from time to time in working with alternating currents. The general theory (general differential equation) of the electric circuit is not dealt with at all. This being so, we of course find no mention of the exponential terms which vanish with time, and which appear in the full solution of the general equation. These, though airily dismissed by many writers, are really of the utmost importance, as on them depends the theoretical treatment of all the important phenomena met with in electric switching, and oscillations set up by sudden changes in the current flowing. These exponential terms certainly constitute a "Grundgesetz," and as such should have been mentioned. The work is closed by a table of formulæ, but that greatest sin of omission, no index, is committed.

C. C. G.

\section{THE PRINCIPLES OF DYEING.}

The Principles of Dyeing. By G. S. Fraps, Ph.D., of North Carolina College. Pp. xii +270 ; with 22 illustrations in the text. (New York: The Macmillan Co.; London: Macmillan and Co., Ltd., 1903.) Price $7 s$. net.

T $N$ the preface to this work the author states that "it 1 attempts to apply to the teaching of dyeing the same methods of class-room work, coordinated with experiments in the laboratory, which have proved so successful in the teaching of inorganic chemistry and other branches of science," and its novel feature consists in the insertion, interspersed throughout the text, of a series of experiments, seventy-nine in number, which the student is to carry out in the laboratory.

Although no such division is made by the author, the book may conveniently be considered in two portions, chapters $i$. to vi. giving a general survey of the subject in 53 pages, while the remaining sixteen chapters, occupying 200 pages, are devoted to a systematic amplification.

This larger portion of the book follows the lines adopted in most modern text-books on dyeing, and little need be said in reference to it beyond the obvious remark that, even with the most careful condensation, it is not possible, without dangerous generalisations, to compress into 200 small pages any adequate discussion of the various matters treated under the headings cotton, linen, wool, silk, bleaching, scouring, machinery, general observations, direct cotton colours, basic colours, acid colours, mordant colours, insoluble colours, mercerisation, dyeing of unions, theory of colour, spectrum analysis, dye testing and detection of dyes. The inevitable result of too general statement follows; for example, on p. 25 I the following sentence is found:- "A dye on cloth has nearly the same absorption-spectrum as a solution of the dye of corresponding strength." This is by no means the case, since the hue of the dyed fabric often differs considerably from that of a simple solution of the dye. In the same section a normal spectrum is figured, while the description refers to the prismatic spectrum.

Less importance, however, should be attached to slight errors of statement than to the general scope of the work, and from this point of view the chief interest attaches to the preliminary chapters, to which the author's statement, quoted in the first paragraph, chiefly applies. After a short introductory chapter dealing with the fibres and explanatory of the scheme of the book, the following five sections are each devoted to a study of the composition and characteristics of one of the important groups of dyes, one or two members of each group being used as illustrative of the group. The scheme is well worked out, but sufficient care has not been taken to prevent, what is always a pitfall to students, over generalisation; and instead of giving the student a clear general view of dyeing phenomena, he will probably acquire, by a perfectly logical process, some very erroneous views. For example, chapter vi. is devoted to indigo, chrome yellow, theory of dyeing, and classes of fibres. Now indigo and chrome yellow have absolutely nothing in common, either chemically or in mode of application, and there is not a word of explanation as to the reason for coupling them together until chapter xix. is reached, when it is seen that it is based on the fact that they both form insoluble pigments on the fibre-a purely artificial and altogether insufficient connection.

One would expect, in a book of this type, that the various theories which have been put forward to account for dyeing phenomena would receive considerable attention, but they are not only dismissed in a page and a half, but are quite incorrectly stated.

The experiments detailed in the text are in most cases well chosen, and add greatly to the value of the book, but a student of inquiring mind may well ask why cotton should be dyed with Congo-red in an alkaline bath and wool in a neutral bath, and the results considered as comparative (Exp. 4). Exp. I2 should certainly be modified. It is highly dangerous to tell a student to pour boiling concentrated sulphuric acid into water, even if the word "caution" is interpolated.

This book is very welcome as an obviously original attempt to teach the general principles of dyeing on novel lines, and most of its shortcomings are explainable by the opening sentence in the preface:- "This book is the result of two years' instruction in dyeing."

W. M. G. NO. 1747 , VOL. 67$]$ 